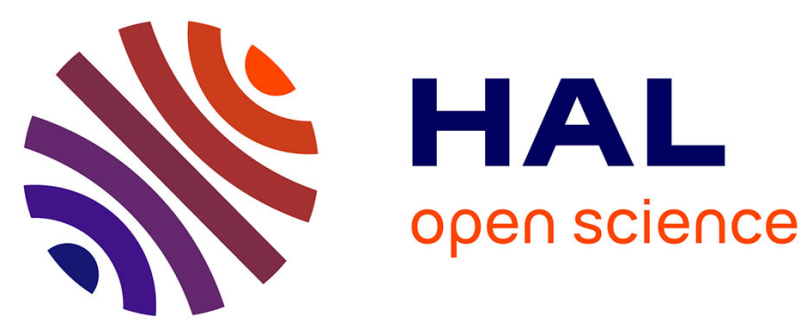

\title{
Electrical assessment of diamond MIM capacitors and modeling of MEMS capacitive switch discharging
}

M Michalas, M. Koutsoureli, Samuel Saada, Christine Mer-Calfati, A. Leuliet, P. Martins, S. Bansropun, P Papaioannou, P. Bergonzo, A. Ziaei

\section{- To cite this version:}

M Michalas, M. Koutsoureli, Samuel Saada, Christine Mer-Calfati, A. Leuliet, et al.. Electrical assessment of diamond MIM capacitors and modeling of MEMS capacitive switch discharging. Journal of Micromechanics and Microengineering, 2014, 24 (11), 10.1088/0960-1317/24/11/115017 . cea01841455

\section{HAL Id: cea-01841455 https://hal-cea.archives-ouvertes.fr/cea-01841455}

Submitted on 3 Feb 2022

HAL is a multi-disciplinary open access archive for the deposit and dissemination of scientific research documents, whether they are published or not. The documents may come from teaching and research institutions in France or abroad, or from public or private research centers.
L'archive ouverte pluridisciplinaire HAL, est destinée au dépôt et à la diffusion de documents scientifiques de niveau recherche, publiés ou non, émanant des établissements d'enseignement et de recherche français ou étrangers, des laboratoires publics ou privés. 


\title{
Electrical Assessment of Diamond MIM Capacitors and Modeling of RF MEMS Capacitive Switches Discharging
}

\author{
L. Michalas, M. Koutsoureli, S. Saada, C. Mer, A. Leuliet, P.Martins, S. Bansropun, G. Papaioannou, \\ P. Bergonzo and A. Ziaei
}

\begin{abstract}
The paper presents the electrical assessment and modeling of the discharge process in RF-MEMS capacitive switches with nanocrystalline diamond dielectric film. The assessment is performed by taking into account the detailed DC electrical characterization of the dielectric film at different temperatures with the aid of Metal-Insulator-Metal capacitors fabricated on the same die. The model assumes screening of trapped charges through carriers that are injected from bottom electrode, transported through grain boundaries and redistributed across diamond film surface through $s^{2}$ state of non diamond carbon. Simulated data and experimental results are found to be in excellent agreement clearly indicating that nanocrystalline diamond can be considered as a MEMS dielectric with predictable discharging process.
\end{abstract}

Index Terms-Diamond, Dielectric Charging, RF MEMS, Reliability, Nanostructure

\section{INTRODUCTION}

$\mathrm{M}$ icroelectomechanical Capacitive Switches (MEMS) have received important research interest over the last two decades mainly due to their successfully incorporation on RF applications such as filters and antennas. Moreover they offer several advantages over the conventional semiconductor counterparts as low insertion loss, low power consumption and high linearity to name a few [1]. Beside that RF MEMS switches are still facing reliability issues, with the most important one being the charging of the solid dielectric film, used to isolate the moving armature from the transmission line. The commonly used dielectrics, low temperature deposited silicon dioxide and silicon nitride, contain a large amount of electrically active defects that may store charges for times longer than $10^{4} \mathrm{sec}$ [2]. The stored charges are responsible for several undesirable effects such as the shift of $\mathrm{C}-\mathrm{V}$ characteristic, narrowing of pull - in/out windows

The paper submitted on $\mathrm{xx}$ June 2014. This work take place within FP7 ENIAC/ESPA-GR project "NANOCOM" under GA: 270701-2, ENIAC call 3 .

L. Michalas, M. Koutsoureli and G. Papaioannnou are with the Physics Department, National Kapodistrian University of Athens, Grecce (e-mail: lmichal@phys.uoa.gr).

S. Saada, C. Mer and P. Bergonzo are with the Diamond Sensors Lab., CEA-LIST, France.

A. Leuliet, P. Martins, S. Bansropun and A. Ziaei are with Thales Research and Technology, France. degradation of $\mathrm{C}_{\mathrm{ON}} / \mathrm{C}_{\mathrm{OFF}}$ ratio and finally device failure due to bridge stiction [3] or shift of pull-in voltage beyond the actuation one.

One of the approaches proposed to overcome the reliability issues related to dielectric charging is the use of alternative dielectric materials, which would exhibit appropriate dielectric permittivity, to provide reasonable high ON/OFF ratio, and drain fast enough the injected charges to obtain the desired device lifetime. In an operating switch, the injected charges that stored during actuation can be removed only via the bottom electrode during the device pull-up state.

Among the proposed alternative dielectric materials diamond has shown very promising features. Studies involving micro/nano crystalline diamond films in MIM (Metal-Insulator-Metal) capacitors revealed suitable discharging behavior [4,5]. In addition RF MEMS capacitive switches with ultra nanocrystalline diamond demonstrated 5-6 orders of magnitude faster recovery time for the bridge release compared to common dielectrics [6]. Moreover the effects of accelerated stress on the pull - in voltage of nanocrystalline diamond switches are reported to be practically insignificant [4]. Finally, MEMS switches have been found to exhibit satisfactory RF performance over a wide frequency range $[7,8]$.

However, despite the reported promising results, there has been no in depth study of the transport properties and their impact on the long term charging and discharging processes in MEMS capacitive switches with diamond dielectric [9], thus the physics behind these processes are still not fully clarified.

The present paper aims is to provide a better understanding of the physical mechanisms relating the long term discharging processes in diamond based RF MEMS capacitive switches and transport properties in the nanocrystalline diamond film. The study employs MIM capacitors and MEMS capacitive switches both fabricated on the same dye. The former are used to assess the material electrical properties through the temperature dependence of the current-voltage characteristics. The discharging processes in MEMS have been monitored through the shift of the bias for minimum up state capacitance [10].

The analysis reveals the features of the long term discharging through the dielectric film while the results are discussed in terms of nanocrystalline material microstructure 
properties. Finally, taking into account the charge transport in the diamond film, the long time discharge process is simulated and compared to experimental results. The excellent agreement between the experimental and theoretical decay indicated that charge transport and screening can considered as responsible for the faster discharge in these devices.

\section{Conduction Mechanisms in Non CRYSTAlline DIAMOND}

Crystalline diamond in its intrinsic condition is an electrical insulator and the only way to increase the conductivity is by doping the film during deposition process. The most common impurity is Boron that leads to p-type conductivity although other impurities have been also implemented [11]. On the other hand amorphous diamond like carbon is quite conductive. Diamond in poly/micro/nano/ultra nano crystalline form is a combination of insulating grains separated by non crystalline carbon areas that provide the conduction paths along the insulating material and are mainly responsible for the film conductivity [12]. From the point of view of physics such areas are characterized by the presence of extended defects that are responsible for the introduction of a large amount of electrically active states and/or zones in the forbidden band gap [11]. Therefore the band gap of micro/nano crystalline diamond is occupied by a practically continuous distribution of states. These are originated by $\pi$ and $\pi^{*}$ states associated to $\mathrm{sp}^{2}$ bonds and distributed symmetrically around Fermi level, $\sigma$ and $\sigma^{*}$ states due to $\mathrm{sp}^{3}$ distorted bonds located also symmetrically around Fermi level at higher energies, thus closer to the band edge, and dangling bonds around midgap as presented in fig.20 of [11].

The origin of $\mathrm{sp}^{2}$ bonds are the non diamond carbon material located at grain boundaries, in contrast to $\mathrm{sp}^{3}$ bonds that are formed by the diamond crystalline structure at the grain areas. As it has been experimentally demonstrated the film conductivity is determined by the ratio of $\mathrm{sp}^{2} / \mathrm{sp}^{3}$ bonded carbon while the film structure is usually revealed by the characteristic peaks obtained in a Raman spectra [12,13].

The electrical charges are able to move across the material via the highly conductive $\mathrm{sp}^{2}$ related states even at relatively low temperatures or applied fields. In the regime of low applied fields and low temperatures the conduction mechanism is thermally activated arising from hopping across $\mathrm{sp}^{2}$ related states and obeying either Mott's law or Arrhenius dependence with low activation energy around $0.1-0.2 \mathrm{eV}$. At higher temperatures the film conductivity is also thermally activated. In this case the reported typical activation energies usually are in the range of $0.7-1 \mathrm{eV}$ or even higher. Such activation energies have never been reported on single crystal films and therefore are attributed to charges generated by thermal activation via gap states associated with $\mathrm{sp}^{2}$ bonds and therefore to the non diamond carbon material at grain boundaries [14]. The $\mathrm{sp}^{2}$ related states contribute also to charge conduction across grains surface. Specifically it has been reported that the surface conductivity of grain boundary regions is higher than that of grain plane regions, which provides experimental evidence that the surface conductivity of diamond films is partly depending on the grain boundaries and that the local surface conductivity increases with the increase in the number of grain boundaries existing in this area [15].

Moreover, parameters such as deposition conditions and/or grain size or grain boundary area and hydrogenation are in principle related to the increase/decrease the ration of $\mathrm{sp}^{2} / \mathrm{sp}^{3}$ bonded carbon [13] and therefore affect the film conductivity. In addition at high fields and temperatures an additional term in conductivity may emerge, which is related to the contribution of the conductivity from the grain areas of the film $[16,17]$.

\section{EXPERIMENTAL}

The tested devices are bridge type RF MEMS capacitive switches with $450 \mathrm{~nm}$ nanocrystalline diamond dielectric film. The nanocrystalline diamond films has been deposited by Microwave Plasma assisted Chemical Vapor Deposition (MPCVD) on $\mathrm{Si}(100) / \mathrm{TiW} / \mathrm{Au}$ substrate. Plasma treatments were performed in a home made designed MPCVD reactor equipped with a $2.45 \mathrm{GHz}-2 \mathrm{~kW}$ SAIREM microwave generator. The base pressure inside the chamber was about $10^{-}$ ${ }^{9}$ mbar. High nucleation density is needed for the early stage of diamond growth in order to form rapidly a continuous diamond film. Nanoseeding technique [18], which consists in the deposition of diamond nanocrystals by spin coating on the substrate, was used to obtain crystal density higher than $10^{11}$ $\mathrm{cm}^{-2}$. Then synthesis of the nanocrystalline diamond film with columnar structure was performed by MPCVD with a mixture of methane $\left(\mathrm{CH}_{4}\right)$ at $0.6 \%$ diluted in hydrogen $\left(\mathrm{H}_{2}\right)$. The total pressure, total gas flow rate, and microwave power during the growth were maintained at $35 \mathrm{mbar}, 250 \mathrm{sccm}$, and $900 \mathrm{~W}$ respectively. During growth the film thickness is monitored by a home-made laser interferometry system [19]. This system is used to stop the experiment at the desired thickness. The surface morphology has been characterized by SEM (Fig. 1) and AFM images that reveal the nanocrystalline structure with a grain size of about $90 \mathrm{~nm}$.

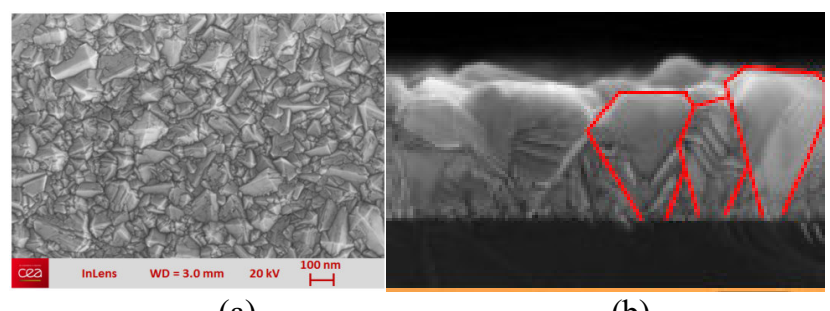

(a)

(b)

Fig. 1 SEM of nanocrystalline diamond used in both MIM capacitors and MEMS capacitive switches, (a) top view and (b) and cross section

The MEMS capacitance voltage characteristics have been obtained by a Boonton $72 \mathrm{~B}$ offering $\mathrm{fF}$ resolution while the applied bias provided by the voltage source of a Keithley 6487. The discharging processes were monitored by recording the shift of the bias for minimum up state capacitance as obtained by the continuously recording of the $\mathrm{C}-\mathrm{V}$ 
characteristics [10] in the up-state. In principle the bias at which the up-state capacitance attains minimum $\left(\mathrm{V}_{\min }\right)$ is the one for which the electrostatic force becomes minimum independently of the presence of bridge creep. Assuming that the deformation of moving armature in the up-state is minimal then the relation between $\mathrm{V}_{\min }$ and the net charge density $\mu_{\psi}$ at the surface of the dielectric film is given by $[3,10]$ :

$$
V_{\min }=\frac{d_{\varepsilon}}{\varepsilon_{r} \varepsilon_{0}} \cdot \mu_{\psi}
$$

The charging was performed by stressing the switch under electric field intensities of $600 \mathrm{KV} / \mathrm{cm}$ and $1 \mathrm{MV} / \mathrm{cm}$ for 300 $\mathrm{sec}$, which correspond to applied bias of $\mathrm{V}_{\mathrm{pi}}$ and $1.6 \mathrm{~V}_{\mathrm{pi}}$ respectively.

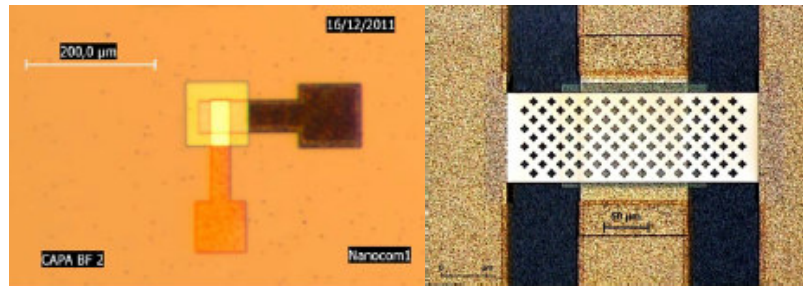

(a)

(b)

Fig. 2 Diamond MIM capacitors (a) and RF MEMS capacitive switches (b)

The current-voltage characteristics of MIM structures, which have been fabricated on the same chip in order the nanocrystalline diamond film to exhibit the same characteristics, have been recorded in the temperature range of $200 \mathrm{~K}$ to $400 \mathrm{~K}$. Finally, all measurements have been performed in vacuum after annealing at $140^{\circ} \mathrm{C}$, in order to minimize any interference from humidity.

\section{Results AND Discussion}

The charging mechanism in MEMS capacitive switches has been shown to occur under the presence of high electric field, above pull-in, through Trap-Assisted-Tunneling and charge redistribution through different transport mechanisms such as the Frenkel-Poole one $[20,21,22]$. On the other hand the discharge process takes place under relatively low electric fields, below pull-out, and the injected and trapped charges are collected by the bottom electrode through hopping mechanism [2].

These clearly imply that the in depth analysis of charging and discharging mechanisms in MEMS capacitive switches requires the understanding of conduction mechanisms in the dielectric film, which can only be achieved by assessing both MIM capacitors and MEMS capacitive switches.

\section{A. Assessment of MIM Capacitors}

The current-voltage characteristics of MIM capacitors provide information on the conduction mechanisms under the presence of low and high electric fields, hence during discharging for charge collection and charging for the redistribution of injected charges, respectively. The typical current voltage characteristics of the diamond MIM capacitors recorded in a loop mode are presented for selected temperatures in the range of $200 \mathrm{~K}$ to $400 \mathrm{~K}$ in Fig. 3.

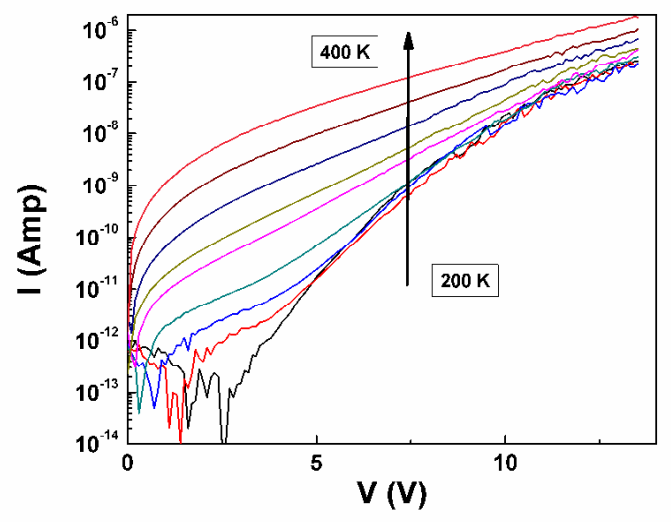

Fig. 3 I-V characteristics of MIM structures have been recorded up to $300 \mathrm{KV} / \mathrm{cm}$ and in the temperature range of $200 \mathrm{~K}$ to $400 \mathrm{~K}$.

The identification of the charge transport mechanisms across a dielectric film is obtained from the current voltage characteristics and the corresponding signature plots. Typical current-voltage characteristic obtained at $300 \mathrm{~K}$ is presented in Fig. 4.

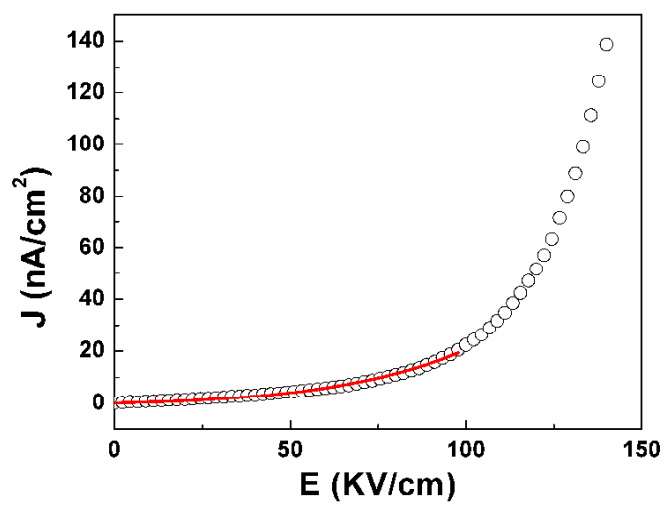

Fig. 4 Non linear fitting with Eq. (2) on the experimental data confirms hopping conductivity for applied field intensities below $100 \mathrm{KV} / \mathrm{cm}$ at $300 \mathrm{~K}$.

As already mentioned, hopping transport dominates at low electric fields. Thus, the hopping conduction equation:

$$
J(E)=\sigma_{H} \cdot E \cdot \exp (\alpha \cdot E)
$$

has been fitted to experimental data of Fig. 4 in the range 0$100 \mathrm{KV} / \mathrm{cm}$. In Eq. (2) $\sigma_{\mathrm{H}}$ is the corresponding hopping conductivity, $\mathrm{E}$ the applied electric field intensity and $\alpha$ is a constant related to material microstructure characteristics [23]. As presented in Fig. 4, equation (2) fits well to the experimental data for field intensities below $100 \mathrm{KV} / \mathrm{cm}$.

Although the above presented fitting results provide a 
strong support for hopping conductivity a deeper investigation is required in order to conclude on the conduction mechanism under low field. This is because the majority of conduction mechanisms in dielectrics show an exponential dependence of current on the applied electric field [24]. Therefore in order to identify the mechanism responsible for the low field transport across the diamond film, the current dependence on temperature under constant electric field has been also investigated [5]. The corresponding signature plot provides a stronger support since different exponential conduction mechanism obeys different temperature dependences. In a hopping system the current dependence on temperature under constant electric field usually obeys the Mott's law I $\sim \exp [-$ $\left.\left(\mathrm{T}_{0} / \mathrm{T}\right)^{1 / 4}\right]$, or exhibits Arrhenius dependence with activation energy $\mathrm{E}_{\mathrm{A}}$ in the range of $0.1-0.2 \mathrm{eV}$ for diamond, $\mathrm{I} \sim \exp (-$ $\mathrm{E}_{\mathrm{A}} / \mathrm{kT}$ ). The latter supports also the contribution of carrier thermal generation, which in a disordered material with relatively high density of states in the band gap may be present even at room temperature.

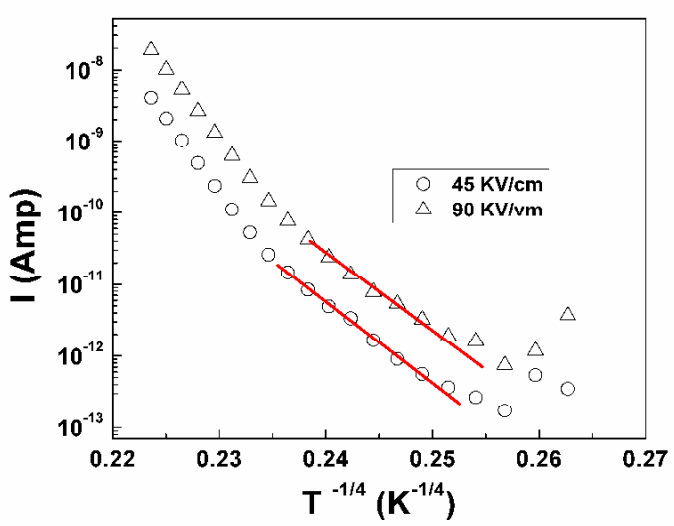

(a)

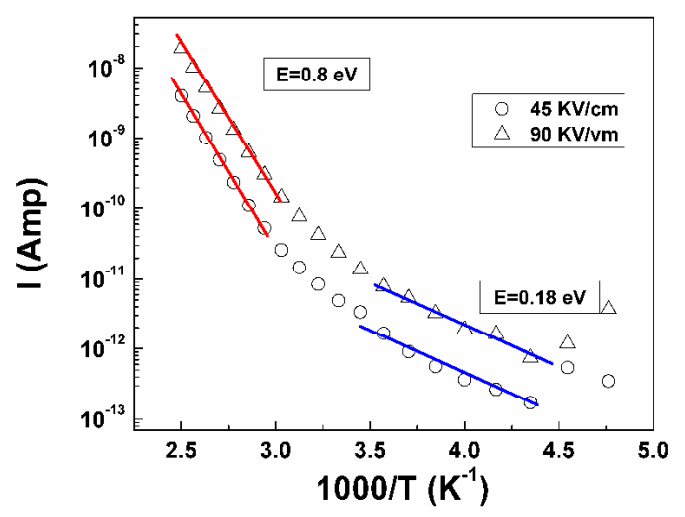

(b)

Fig. 5 Signature plots for hopping conductivity at low fields. At lower temperatures the data obey well both Mott's (a) and Arrhenius' (b) law, both denoting hopping.

The linear regime presented in Fig. 5a denotes that the current conduction obeys the Mott's law, $\mathrm{I} \sim \exp \left[-\left(\mathrm{T}_{0} / \mathrm{T}\right)^{1 / 4}\right]$ for hopping conductivity. Such dependence has been obtained in diamond like carbon films [25] denoting that under low electric field the conductivity take place through hopping in non diamond carbon material located, in the present case, at the grain boundaries. In addition the low temperature regime can be also fitted by an Arrhenius dependence with activation energy $\mathrm{E}=0.18 \mathrm{eV}$ as presented in Fig. 5b. Such behavior also supports hopping associated at the grain boundaries areas $[14,26]$. Moreover the activation energy obtained for the higher temperature regime, $\mathrm{E}=0.8 \mathrm{eV}$ is typical of thermal generation through gap states associated to non diamond carbon material, which is located at grain boundaries [14,27].

Thus, the low field conductivity in the MIM dielectric films is determined by the properties of the amorphous diamond like carbon areas surrounding the diamond grains and therefore the latter area is expected to have the major role on the discharging behavior.

\section{B. Assessment of RF MEMS switches}

In order to investigate the discharging process in RF MEMS capacitive switches, the devices have been stressed under electric field intensities of $600 \mathrm{KV} / \mathrm{cm}$, correspond to a condition just above pull-in and under $1 \mathrm{MV} / \mathrm{cm}$, which is a commonly observed applied field for RF MEMS, for time duration of 300 seconds. The net surface charge after each stressing cycle has been calculated from the shift of minimum up-state capacitance voltage characteristic with respect to the non stressed one using Eq. 1. Typical shift caused by stressing the MEMS dielectric film is presented in Fig. 6. The calculated voltage shift $\Delta \mathrm{V}_{0}$ and the corresponding stored charge for each stressing field are summarized in Table 1.

Assuming that the injected charges are confined close to the injection electrodes [21,22], the generated field can be approximated by:

$$
E=\frac{V_{\text {min }}}{d}
$$

In principle, this field intensity determines the discharging process during the charge collection through the bottom electrode. The charge transport and collection is expected to take place through sequential trapping and emission, percolation etc that macroscopically obey the stretched exponential law.

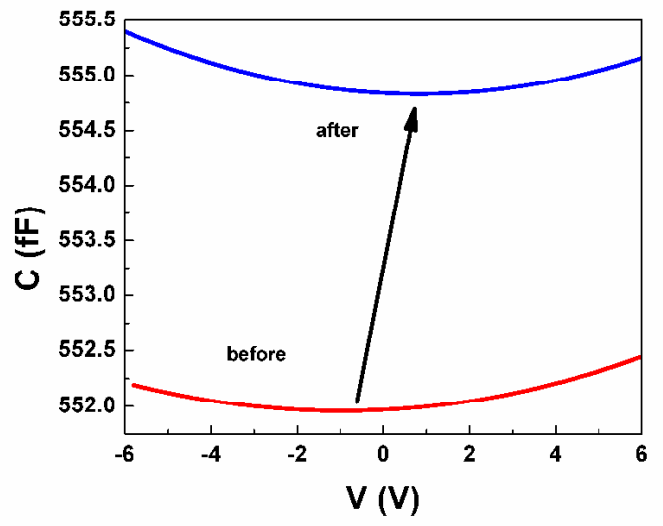

Fig. 6 Shift of up-state C-V characteristic due to charging. 
TABLE 1. Stored Charge as a function of stressing field

\begin{tabular}{|c|c|c|}
\hline $\begin{array}{l}\text { Field Intensity } \\
(\mathrm{KV} / \mathrm{cm})\end{array}$ & $\Delta V_{0}(V)$ & $\begin{array}{c}\text { Stored charge } \\
\left(\mathrm{nC} / \mathrm{cm}^{2}\right)\end{array}$ \\
\hline 600 & 0.9 & 9.7 \\
\hline 1000 & 2.2 & 24 \\
\hline
\end{tabular}

In order to get a deeper insight it is essential to take into account that the charging process is determined by the film microstructure. The space charge results from simultaneous actions of charge injection from the metallic electrodes via trap assisted tunneling (TAT) and assisted by the field emission process in non contacting areas [28,29] and the charge redistribution through Poole Frenkel emission of the trapped charges under the high electric field (Table 1) during actuation. Thus the charge spatial distribution will be determined by the density of states at the surface and bulk of the dielectric film [21].

The long term discharging process has been monitored by continuous recording of the shift of bias for minimum up state capacitance up to 1800 seconds. A typical discharging behavior is presenting in following Fig. 7 .

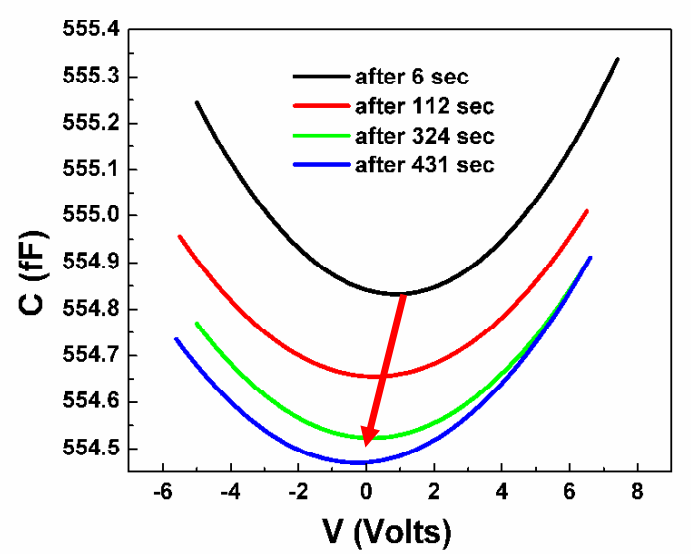

Fig.7 Evolution of up-state $\mathrm{C}-\mathrm{V}$ characteristic during discharging process

The experimentally obtained discharging decay for the different stressing fields is presented in addition to the theoretical calculation based on the proposed model discussed in the next session in Fig. 8(a) for stressing field of $600 \mathrm{KV} / \mathrm{cm}$ and in Fig. 8(b) for the case of $1 \mathrm{MV} / \mathrm{cm}$. The analysis revealed exponential relaxation $(\beta=1)$ for the low field case and a stretched exponential $(\beta<1)$ one for the more complicated condition that is built up by the higher charging field in case of $1 \mathrm{MV} / \mathrm{cm}$. It also important to point out that the time constant $(\tau)$ obtained by the fitting procedure is in the range of $250 \mathrm{sec} \tau \mathrm{o} 300 \mathrm{sec}$ (Table 2) while in both cases the discharging process were practically completed after about $1000 \mathrm{sec}$. Considering that the corresponding discharging time for $\mathrm{SiN}_{\mathrm{x}}$, which is the typical dielectric material for $\mathrm{RF}$ MEMS, is at least 10 times longer [2], the latter are quite important results supporting the use of diamond films as alternative dielectric for RF MEMS.

\section{Modeling and discharging process}

The study of the electrical characteristics of MIM capacitors (section A) provides information on the conduction mechanisms responsible for the discharging behavior of the RF MEMS capacitive switches. Due to the low electric field intensity the signature plots of the conductivity are attributed to hopping associated with the $\mathrm{sp}^{2}$ bonds located at grain boundaries and film surface. Taking these into account as well as the conduction mechanism across the surface of grains [15], as already mentioned above, a potential mechanism is proposed for the fast discharging observed in diamond based RF MEMS. The mechanism assumes compensation of trapped charges through screening from charges injected from the bottom electrode and transported and redistributed across the surface through the $\mathrm{sp}^{2}$ states. Such an assumption allows the direct use of the parameters of charge transport through hopping mechanism. The hopping parameters of Eq. 2 are $\sigma_{\mathrm{H}}$ $=1.2 \times 10^{-15}\left(\mathrm{Scm}^{-1}\right)$ and $\mathrm{a}=5.8 \times 10^{-6}(\mathrm{~cm} / \mathrm{V})$ and have been extracted from the I-V characteristic of MIM capacitors.

In a MEMS capacitive switch, the discharge current through the insulating film is given by [10]:

$$
J_{\text {dis }}(t)=-\frac{d \mu_{\psi}(t)}{d t}
$$

Taking into account Eq. (1) and Eq. (3) for the average internal electric field in the film, we are led to:

$$
\frac{d E(t)}{d t}=\frac{\sigma_{H}}{\varepsilon_{r} \varepsilon_{0}} \cdot E(t) \cdot \exp (\alpha \cdot E(t))
$$

the integral of which provides a relation between the value of electric field E and time during "discharge" through the insulating film:

$$
t=-\frac{\varepsilon_{r} \varepsilon_{0}}{\sigma_{H}} \int_{E_{0}}^{E(t)} \frac{d E}{E \exp (\alpha E)}=-\frac{\varepsilon_{r} \varepsilon_{0}}{\sigma_{H}} \int_{V_{\min , 0}}^{V_{\min }(t)} \frac{d V_{\min }}{V_{\min } \exp \left(\frac{\alpha V_{\min }}{d}\right)}(6)
$$

where $E_{0}$ and $V_{\min , 0}$ are the initial internal field and bias for minimum up-state capacitance respectively, $\mathrm{E}(\mathrm{t})$ and $\mathrm{V}_{\min }(\mathrm{t})$ are the corresponding values at time t. Here it is important to point out that even if the values of $E_{0}$ and $V_{\min , 0}$ are not experimentally determined, due to delay $\Delta \mathrm{t}$ introduced by the experimental setup, Eq. (6) can be still applied taking into account that $\mathrm{V}_{\min , 0}$ has to be replaced with $\mathrm{V}_{\min , 1}$, the first measured value, and the left hand side term will represent the time with offset $\Delta \mathrm{t}$.

The simulation of discharge process in a MEMS capacitive switch, with nanocrystalline diamond dielectric film, based on the proposed model and the data extracted from MIM currentvoltage characteristics is presented in Fig. 8 for stress conditions discussed in section B. Moreover, taking into account that the discharge process is rather complex, the stretched exponential relaxation was also fitted to experimental data in order to provide parameters that are easily introduced in simulation tools but do not provide insight on the physical processes involved. 


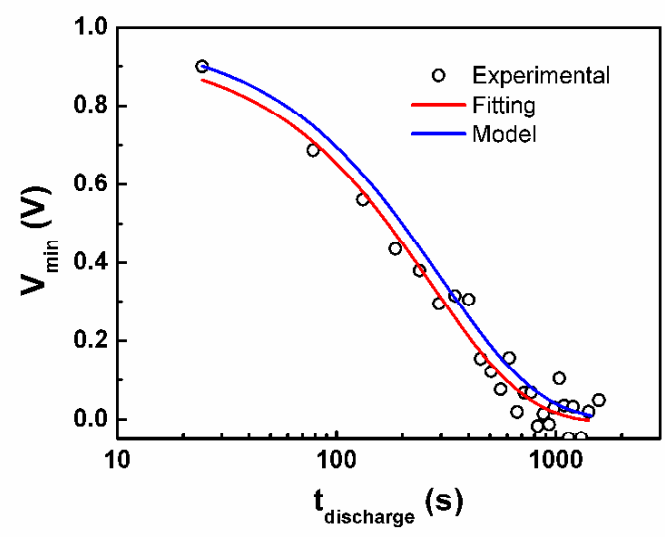

(a)

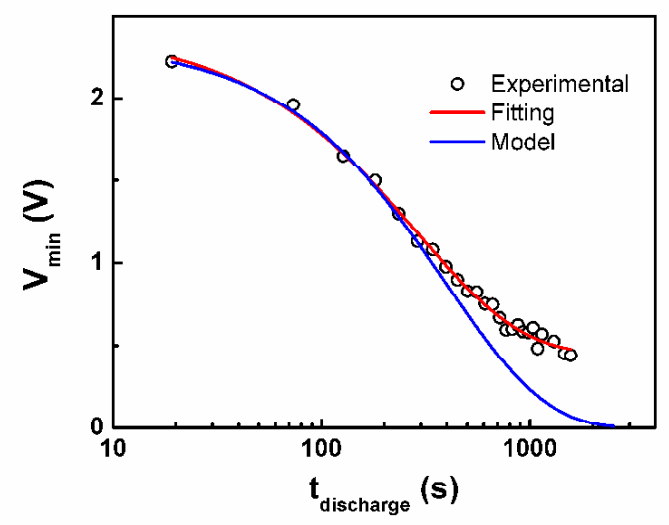

(b)

Fig. 8 Experimental results with fitting procedure and model predictions for the discharging process of MEMS after charging with (a) $600 \mathrm{KV} / \mathrm{cm}$ and (b) $1 \mathrm{MV} / \mathrm{cm}$

TABLE 2. Fitting results on the experimental data

\begin{tabular}{|c|c|c|}
\hline $\begin{array}{c}\text { Charging Field } \\
\text { (KV/cm) }\end{array}$ & $\mathbf{T}$ (sec) & $\boldsymbol{\beta}$ \\
\hline 600 & 270 & 1 \\
\hline 1000 & 285 & 0.87 \\
\hline
\end{tabular}

The fitting results, summarized in Table 2, revealed Debye relaxation for the lower stress level and a more complex one for the higher one.

Regarding the plots, an excellent agreement between the experimental results and the model predictions is obtained in case of charging under $600 \mathrm{KV} / \mathrm{cm}$ (Fig 8(a)). In case of charging under $1 \mathrm{MV} / \mathrm{cm}$ (Fig 8(b)) the simulation deviates from experimental data which show a slower discharge rate. Such behavior may be attributed to contribution of a slower discharging mechanism in this case supported also by the reduction of $\beta$ factor in the stretched exponential fitting. Other charging effects that may contribute to this behavior can be related to charging due to handling or substrate dielectric material charging [30].

\section{CONCLUSIONS}

The mechanisms responsible for the discharging process in RF MEMS capacitive switches with nanocrystalline diamond dielectric film have been in depth investigated. MetalInsulator-Metal capacitors have been employed for the study of the dependence of transport mechanisms on electric field intensity and temperature. The signature plots obtained from the current-voltage characteristics denoted that under low electric field intensities the charge transport across the nanocrystalline film takes place through hopping across the non diamond carbon material located mainly at the grain boundaries and film surface. Based on this, a discharge model has been proposed which is assumed to rise from compensation through screening with charges injected from the bottom electrode and transported and redistributed across the surface through the $\mathrm{sp}^{2}$ states at grain boundaries.

Employing the transport parameters obtained from MIM capacitors, the proposed model was used to simulate the discharge process of MEMS capacitive switches. The comparison of the simulation data to experimental ones showed excellent agreement for the higher charging levels. This is of significant importance because the model allows the prediction of recovery of a MEMS capacitive switch with nanocrystalline diamond dielectric film. The deviations observed at the low charging levels, tails, can be attributed to substrate charging.

Finally, it is worth mentioning that the discharging characteristics as predicted and recorded support the use of nanocrystalline diamond as alternative dielectric to enhance the lifetime of RF MEMS capacitive switches.

\section{ACKNOWLEDGMENT}

The authors would like to acknowledge that the present work has been supported by the FP7 ENIAC/ESPA-GR project "Microsystem Based on Wide Band Gap Materials Miniaturized and Nanostructured RF-MEMS" NANOCOM under GA: 270701-2, ENIAC call 3.

\section{REFERENCES}

[1] G.M. Rebeiz, RF MEMS: Theory, Design and Technology, New York: J. Wiley \& Sons, 2003.

[2] M. Koutsoureli, L. Michalas and G. Papaioannou"Charge collection mechanism in MEMS capacitive switches" in Proc. IEEE Int. Reliab. Phys. Symp., pp. ME2.1- ME 2.5, April 2012.

[3] X. Rottenberg, I. De Wolf, B.K.J.C. Nauwelaers, W. De Raedt, H.A.C. Tilmans, "Analytical model of the DC actuation of electrostatic MEMS devices with distributed dielectric charging and nonplanar electrodes," IEEE J. Microelectromech. Syst., vol. 16, no.5, pp. 1243-1253, Oct 2007.

[4] C. Chen, Y. Tzeng, E. Kohn, C. H. Wang and J.K. Mao, “ RF MEMS capacitive switch with leaky nanaodiamond dielctric film" Diam. and Relat. Mat., vol. 20, pp. 546-550, April 2011.

[5] L. Michalas, M. Koutsoureli, E. Papandreou, G. Papaioannou, S. Saada, S. Mer, H. Regis, P. Bergonzo, A. Leuliet, P. Martins, S. Bansropun and A.Ziaei " Electrical characterization of undoped diamond films for RF MEMS application” in Proc. IEEE Int. Reliab. Phys. Symp., pp. 6B3.16B 3.7, April 2013.

[6] C. Goldsmith, A. Sumant, O. Auciello, J. Carlisle, H. Zeng, J.C.M. Hwang, C. Palego, W. Wang, R. Carpick, V.P. Adiga, A. Datta, C. Gudeman, S. O' Brien and S. Sampath "Charging characteristics of ultra-nano-crystalline diamond in RF MEMS capacitive switches" in Proc. IEEE Int. Microw. Symp. Dig., pp. 1246-1249, Jun. 2010. 
[7] S. Balachandran, D. Hoff, A. Kumar and T. Weller "Nanocrystalline diamond RF MEMS capacitive switch," in Proc. IEEE MTT-S Int Microw. Symp. Dig., pp. 1657-1660, Jun. 2009.

[8] J. Chee, R. Karru, T.S. Fisher and D. Peroulis, "DC-65GHz Characterization of nanocrystalline diamond leaky film for reliable RF MEMS switches" in Proc. $35^{\text {th }}$ Europ. Microw. Conf. pp. 581-584, Oct. 2005.

[9] M.W. Van Spengen, "Capacitive RF MEMS switches dielectric charging and reliability : A critical review with recommendations" J. Micromech. \& Microeng.vol. 22 pp. 074001, 2012.

[10] M. Koutsoureli and G. Papaioannou“ Determination of bulk discharge currents in the dielectric film of MEMS cpacitive switches" Microel. Reliab., vol. 51 pp. 1874-1877, Sept. 2011.

[11] O.A. Williams “ Nanocrystalline Diamond” Diam. and Relat. Mat., vol. 20, pp. 621-640, 2011

[12] T. Sugino, Y. Muto, J. Shirafuji and K. Kobashi "Electrical conduction mechanisms in polycrystalline chemically vapor deposited diamond films" Diam. and Rel. Mat., vol. 2, pp. 797-802, 1993.

[13] E.J. Correa, Y. Wu, J.-G. Wen, R. Chandrasekharan and M.A. Shannon "Electrical conduction in undoped ultrananocrystalline diamond thin films and its dependence on chemical composition and crystalline structure" J. Appl. Phys. vol. 102, 113706, 2007.

[14] E. P. Visser, G.J. Bauhuis, G. Janssen, W. Vollenberg, W.J.P. van Enckevort and L.J. Giling "Electrical conduction in homoepitaxial, boron doped diamond films" J. Phys. Condens. Matter, vol. 4, pp. 7365 7376, 1992.

[15] Y.L. Li, J.J. Li, X.X. Xia, C. Lu, H. Jin and C.Z. Gu "Effect of grain boundary on local surface conductivity of diamond flm" J. Appl. Phys. Vol 105, 013706, 2009

[16] G. De Cesare, S. Salvatori, R. Vincenzoni, P. Ascarelli, E. Cappelli, F. Pinzari and F. Galluzzi "On the electrical properties of polycrystalline diamond films on silicon" Diam. and Relat. Mat., vol. 4, pp. 628-631, 1995.

[17] P. Gonon, Y. Boiko, S. Prawer and D. Jamieson "Poole frenkel conduction in polycrystalline diamond" J. Appl. Phys. Vol 79, no. 7, pp. 3778-3780, 1996.

[18] H. A. Girard, S. Perruchas, C. Gesset, M. Chaigneau, L. Vieille, J.C Arnault, P. Bergonzo, J-P Boilot and T. Gacoin "Electrostatic grafting of diamond nanoparticles: A versatile route to nanocrystalline diamond thin films" Appl. Mat. Interf., vol. 1 pp. 2738 -2746, Nov. 2009.

[19] S. Saada, S. Pochet, L. Rocha, J.C Arnault and P. Bergonzo "Real time investigation of diamond nucleation by laser scattering" Diam. and Relat. Mat., vol. 18, pp. 707- 712, April 2009.

[20] R. Ramprasad " Phenomenological theory to model leakage currents in metal-insulator-metal capacitor systems" Phys. Stat. Sol.(b), Vol. 239 no 1, pp. 59-70, 2003.

[21] G. Papaioannou, F. Coccetti and R. Plana "On the modeling of dielectric charging in RF-MEMS capacitive switches" in Proc. Top. Meet. On Silicon Monolith. Int. Circ. in RF Syst., pp. 108-111, Jan. 2010.

[22] S. Palit, M. A. Alam " Theory of charging and charge transport in "intermediate" thickness dielectrics and its implications for characterization and reliability" J. Appl. Phys. 111, 054112, 2012.

[23] M. Pollak " On dispersive transport by hopping and by trapping" Phil. Mag. Vol. 36, no 5, pp. 1157-1169, 1977.

[24] S.M. Sze, Physics of Semiconductor Devices, New York: J. Wiley \& Sons, 1981.

[25] N. Konofaos and C.B. Thomas "Characterization of heterojuction devices constructed by amorphous diamondlike films on silicon" $J$ Appl. Phys. Vol 81, no. 9, pp. 6238-6245, 1997.

[26] I. Thurzo, D.R.T. Zahn, A.K. Dua, M. Roy and V.C. Geroge "The origin of charge transients in $\mathrm{Al} /$ undoped diamond/p-Si diamond" Diam. and Relat. Mat., vol. 11, pp. 400- 404, 2002.

[27] F. Fontaine and A. Deneuville"High temperature measurments of diamond thin films" Diam. and Rel. Mat., vol. 4, pp. 673-677, 1995.

[28] L. Michalas, A. Garg, A. Venkattraman M. Koutsoureli, A. Alexeenko, D. Peroulis and G. Papaioannou "A study of field emission process in electrostatically actuated MEMS switches" Microel. Reliab., vol. 52 , pp.2267-2271, Sept. 2012.

[29] L. Michalas, M. Koutsoureli, G. Papaioannou “ Probing contactless injection dielectric charging in RF MEMS capacitive switches" IET Electron. Lett., Vol. 50, no 10, pp. 766-768, May 2014.

[30] P. Czarnecki, X. Rottenberg, P. Soussan, P. Ekkels, P. Muller, P. Nolmans, W. De Raedt, H.A.C.. Tilmans, R. Puers, L. Marchand, I. De Wolf "Influence of the substrate on the lifetime of capacitive RF MEMS switches", in Proc. $21^{\text {st }}$ IEEE International Conference on Micro Electro Mechanical Systems, MEMS 2008, pp. 172-175 Jan. 2008.

Loukas Michalas (1980) graduated with a degree in Physics, a M.Sc. in Solid State Physics and the Ph.D. in Solid State Electronics from the University of Athens (NKUA), Greece in 2002, 2004 and 2009 respectively. Since then he works as a research associate at the same university participating in national and EU funded research projects. Main areas of research interest are the electrical properties of semiconductor materials and devices and the dielectric/electrical properties of thin insulating films and nanostructured materials for reliable RF MEMS. He has published 20 articles in international peer reviewed journals and contributed to more than 40 conferences presentations with most of them appearing in reviewed proceedings. He also serves as reviewer for the IEEE and other international scientific publications.

Matroni Koutsoureli graduated from National and Kapodistrian University of Athens (NKUA) in Greece on 2006 where she received the B.Sc. degree in Physics. On 2008 she received the M.Sc. degree and on 2014 the Ph.D. degree in Solid State Physics from NKUA. Since then she works as a research associate in NKUA. Her current research is focused on the polarization effects that appear in thin dielectric films of (RF) Micro-Electro-Mechanical (MEMS) switches. She has published 15 articles in peer reviewed international journals and has contributed to more than 30 conferences presentations.

Samuel Saada Eur.Ing, PhD, HDR, 40, is researcher at CEA with expertise in the field of diamond research. After a $\mathrm{PhD}$ at the Institut National Polytechnique de Lorraine, he joined a high-tech company in 2000 to develop a research program on diamond for microelectronic applications. In collaboration with CEA, he designed a unique research system dedicated to diamond growth and connected to an Ultra High Vacuum characterization platform. He joined the CEA company in 2006 and participate to many research programs for innovative diamond applications. His research area concerns diamond nucleation and growth, heteroepitaxy, plasma/surface interactions, CVD process, etc. He is author of over 50 contributions in scientific journals.

\section{Christine Mer}

Aude Leuliet received her PHD on "Simulation of electronic transport in Quantum Cascade Lasers" from University Paris 7 in 2010. She is working on the reliability of RF-MEMS devices at Thales Research and Technology (France). Her current research activities are focus on the study of dielectric electrical properties and electronic transport simulation in relation with her RF-MEMS activities.

Paolo Martins (1979) graduated with a Master degree in Micro and Nanotechnologies from University of Claude Bernard Lyon (FR) in 2005. He obtained his Ph.D. on mechanical characterization of submicron thin films for MEMS and NEMS applications from the National Institute of Applied Sciences of Lyon (FR) in 2009.In 2009, he moved to Thales Research and Technology as research engineer where 
he is responsible to the RF MEMS technology development. His current research focuses on the technological aspects and innovative approach in the field of RF MEMS applications. He has co-authored in several publications and patents and he is involved in several European projects (MEGA, SATURNE, MINIMEMS, NANOTEC, NANOCOM ...).

Shailendra Bansropun (1974) graduated with a Bachelor degree in electronics/telecommunications and a Ph.D. in heterojunction bipolar transistors from the University of Sheffield (UK) in 1996 and 2001 respectively. Following a couple of years of engineering and design experience in the free space optics and telecommunication industry, he then moved on to Thales Research and Technology where he currently holds the post of research and technology engineer within the common technological platform in Palaiseau, France. His current research focuses on the technological aspects and innovative approaches in the various fields covering photonic devices, quantum dots devices, subwavelength diffractive optics as well as MEMS devices among others. He has co-authored over 25 publications and has also been involved in several European projects( BIGBAND, TERANOVA, KORRIGAN,ANSWER).

George Papaioannou received a B.Sc. degree in physics from University of Athens, and M.Sc. degree from University College London, England, and a Ph.D. degree in Solid State Physics from University of Athens. Presently he is with the Solid State Physics Section of Athens University, leading a team on the transport properties and the radiation effects in compound semiconductors and devices and the interaction of light in III-V compound semiconductors and SOI structures and devices. Recently, he is working on the investigation of charging mechanisms in insulating materials of RF-MEMS switches. He has contributed to 125 publications and 162 conference presentations.

Dr Philippe Bergonzo Eur.Ing, PhD, HDR, 45, is Research Director at CEA with expertise in the field of diamond research. After a $\mathrm{PhD}$ at University College London UK, he Joined CEA in 1994 to focus on novel material development for device fabrication. Since 2003, he took the lead of a team now called the Diamond Sensors Laboratory. The team developments address novel tools taking advantage on diamond exceptional properties from thermal management to detection properties, via electrochemical applications and bioand chemical sensor developments. The lab also aims at novel sensors (SAWs and MEMS), to bio-interfaces (cells and retinas), to diamond nanoparticles for drug delivery applications and electrochemical industrial applications. He is author of over 200 contributions in scientific journals and is a member of several diamond conference committees and editorial scientific committees.

Afshin Ziaei received the B.S. degree in Electronics Engineering from the University of Paris XI (Orsay) in 1997 and the M.S. degree in Electronics and Microwaves Engineering from the University of Paris VI (Jussieu) in 1998 and he received Ph.D. degree in microwave from the Department of Electronics Engineering at CNRS-IEMN
(Institut d'Electronique et de Microélectronique et de Nanotechnologie) in 2001. He joined the research engineer at Thales Research \& Technology, France, in 1999. His research interests include the development, design, fabrication, and characterization of RF MEMS (Micro Electro Mechanical Systems) components such as mechanical switches, transmission lines, etc. He was involved or in charge of many French or European Projects (such as ARHMS, NANOPACK (coordinator of projects), ARESSAT, MEMS2, PEA MEMS, FLAME). He is a member of IEEE. 